In Cres. Vol. $5 N^{\circ}$ 1: pp. 113-130, 2014

\title{
REVISTAS DE LITERATURA INFANTIL Y JUVENIL EN EL PERÜ*
}

\author{
MAGAZINES OF INFANTILE AND JUVENILE \\ LITERATURE IN PERU
}

Saniel E. Lozano Alvarado

\begin{abstract}
RESUMEN
En el presente texto se ofrece un panorama nacional sobre la publicación de revistas de literatura infantil en el Perú desde el siglo XIX hasta la actualidad. Se puede apreciar que esta actividad se realiza en varias ciudades y regiones del país, aunque, por lo general, no se trata de un proceso continuo, de periodicidad regular, sino que está expuesto a una serie de factores que no siempre favorecen una edición permanente. El propósito general que anima la edición de estas publicaciones es promover la difusión del libro y la lectura entre los niños y jóvenes, estimular la creatividad, difundir experiencias didácticas, difundir la publicación de nuevos libros y apoyar la educación. El trabajo está dirigido especialmente a los educadores, niños, jóvenes, escritores, editores, artistas gráficos y padres de familia.

PALABRAS CLAVE: Revistas, edición, literatura infantil y juvenil.
\end{abstract}

\section{ABSTRACT}

In this text provides a national overview of magazine publishing children's literature in Peru since the nineteenth century to the present. It can be seen that this activity is carried out in several cities and regions, although usually it is not a continuous, regular basis, but is exposed to a number of factors that are notal ways in a permanent edition. The general purpose animating editing these publications is to promote the dissemination of books and reading among children and young people, stimulate creativity, disseminate learning experiences, disseminate the publication of new books and support education. The work is particularly aimed at educators, children, youth, writers, editors, graphic artists and parents.

KEY WORDS: Magazines, publishing, children's literature.

* Recibido: 14 de febrero del 2014; aprobado: 10 de junio del 2014.

1 Profesor de la Universidad Privada Antenor Orrego, Trujillo - Perú. Director de "Rayuelo", revista oficial de la Asociación Peruana de Literatura Infantil y Juvenil (APLIJ). 


\section{INTRODUCCIÓN}

En el afán permanente de varios escritores, maestros, periodistas, artistas gráficos, por llevar el libro y la lectura a los niños y jóvenes, así como en la tarea de promover la sensibilidad y el desarrollo de la creatividad, se han publicado varias revistas, especialmente a partir de la segunda década del siglo XX, aunque los antecedentes más lejanos hay que encontrarlos, en realidad, mucho antes, incluso en el siglo anterior.

Sin embargo, en una apreciación de conjunto, los signos dominantes de las revistas de literatura infantil en el Perú es su poca vigencia y permanencia; su publicación eventual y circunstancial; su periodicidad irregular. Muchas aparecen pletóricas de emoción, entusiasmo y expectativa; a veces alcanzan cierta continuidad, pero por poco tiempo; otras aparecen, desaparecen y reaparecen. Además, el contenido de la mayoría, como tiene que ser, está constituido por textos de creación de los propios niños y de los escritores adultos ganados por la inspiración o el propósito de contribuir a la formación de los niños y jóvenes. Muy pocas incluyen textos de teoría, ensayo, análisis y crítica literaria, especialmente en el área respectiva. También están ausentes los enfoques didácticos y metodológicos o las muestras de experiencias, propuestas y planteamientos sobre la enseñanza de la literatura a los niños y jóvenes. Por lo demás, es natural que esto ocurra así, toda vez que este tipo de publicaciones tienen un público específico interesado por la literatura de creación, especialmente el cuento, la poesía, la tradición oral. Asimismo, varias de estas revistas no se limitan al contenido o tema netamente literario, sino también desarrollan asuntos conexos, como historia, geografía, folclore, ciencia, manualidades, entretenimientos, etc.

En esta exposición nos proponemos ofrecer un panorama de las revistas de literatura infantil y juvenil publicadas en el país.

\section{ANTECEDENTES}

"El Atomo". Hasta donde tenemos conocimiento, es la publicación más antigua sobre el asunto. Apareció en Trujillo, en la fecha central de nuestra efemérides nacional: el 28 de julio de 1888. Fue la primera revista ilustrada; mejor diríamos, el primer periódico, pues se editó en formato tabloide. Aunque no se consignaba expresamente el nombre del director, sino sólo la referencia: Redacción anónima; Administrador: Julio Martínez Serrano, que curiosamente, fue un médico ecuatoriano que residía en la ciudad por haber sido desterrado 
de su país. El desarrolló una labor pionera, diversa y múltiple, pues fue el director, dibujante y autor de los grabados, que entonces se confeccionaban en madera; el zinc, para los clisés vendría posteriormente. Los destinatarios principales eran los niños; por tanto, históricamente es el primer medio periodístico del país que marca los antecedentes de la literatura infantil y juvenil en el Perú, incluso, después del título aparecía el lema, a manera de subtítulo: "Periódico infantil ilustrado". El propio contenido estaba constituido por temas directamente relacionados con la formación de la niñez; por eso es muy visible la orientación didáctica y educativa de los diversos temas. Dicho periódico dividía su contenido en dos grandes secciones: Sumario y Grabados, cuya ubicación aparecía siempre en la primera página.

Es del todo sorprendente la aparición y edición de un medio periodístico dedicado a la infancia, en una época en que los principales acontecimientos nutrían casi por entero las páginas de la naciente prensa peruana. Claro que los textos incluidos no pertenecen a ningún autor peruano, lo cual es comprensible en una época en que la cultura y la literatura nacional siguen los parámetros foráneos, especialmente europeos. Por eso, tal actitud no es ningún demérito; todo lo contrario, si se tiene en cuenta que la cultura, en general, no tiene fronteras, pues "El Átomo" se nutrió de los contenidos culturales de la época.

Se trata de un periódico especializado prácticamente sin antecedentes, por lo menos en nuestro país. En tal sentido, el ecuatoriano Julio Martínez Serrano, que fue director, administrador, diseñador, ilustrador, editor, es el auténtico pionero de las revistas y periódicos de literatura infantil y juvenil en el Perú.

No se sabe exactamente el periodo de duración; pero el acucioso investigador Jorge Kishimoto, notable coleccionista especialmente de la producción literaria de César Vallejo, a quien debemos esta información, ha elaborado la siguiente relación de los primeros diez ejemplares, cuyo contenido resumimos a continuación.

\section{$\mathrm{N}^{\circ} 1: 28$ de julio de 1888.}

SUMARIO: 28 de Julio.- Recomendación.- Mis grabaditos.- Lección de amor: poesía.- Viajes de Gulliver a Lilliput.- La Manta.-Arca de Noé.

GRABADITOS, consistente en dibujos, ilustraciones de los temas y artículos, comentarios y opiniones.

\section{$\mathrm{N}^{\circ}$ 2: 4 de agosto de 1888. Semanario Infantil Ilustrado.}

SUMARIO: Mañana .- La Rana y el Renacuajo (fábula).- Mis grabaditos.Viajes de Gulliver a Lilliput.- Arca de Noé.

GRABADOS: El baño de la muñeca, etc. 
En el segundo número se reprodujo de "El Perú Ilustrado", de Lima, el siguiente comentario, que constituyó todo un respaldo a la aislada iniciativa:

"El Atomo".- Nos ha visitado ya el primer número de este estimabilísimo coleguita. Buen material y buenos grabados. trae "El Atomo", que á pesar de su título y su diminuto cuerpo, se manifiesta valiente adalid del progreso y alegre amigos de esos tiernos seres, sobre quienes pesarán mañana, iguales deberes que los que nosotros hoy, apenas si sabremos llenar ámedias.

Ya hemos recomendado á los padres de familia, á este nuestro hermano menor. Volvemos á hacerlo ahora, aunque el nene no necesite recomendación ninguna.

También la sección permanente, “Arca de Noé”, traía ilustrativos pensamientos y máximas, como las siguientes:

La conciencia es á la vez

Testigo, fiscal y juez.

$* * *$

Sin virtud la ciencia humana

Es caña frágil y vana.

$* * *$

Si es bueno y dócil un niño,

De todos gana el cariño.

\section{$N^{\circ}$ 3: 11 de agosto de 1888.}

\section{$N^{\circ} 4: 18$ de agosto de 1888 .}

TEXTO: ¡Desobediente! - Mis grabaditos.- Amor filial (poesía).- El Premio (fábula).- Viajes de Gullivert a Lilliput.- Arca de Noé.

GRABADOS.

\section{$N^{\circ} 5: 25$ de agosto de 1888 .}

SUMARIO.

TEXTO: El perezoso y el madrugador.- Mis grabaditos.- Cuento infantil.Viajes de Gulliver.- La fiesta de cumpleaños.

GRABADITOS.

\section{$\mathrm{N}^{\circ}$ 6: 10 de setiembre de 1888.}

SUMARIO.

TEXTO: La fiesta de cumpleaños.- Mis grabaditos.- Viajes de Gulliver.Arca de Noé.

GRABADOS. 


\section{$\mathrm{N}^{\circ}$ 7: 7 de setiembre de 1888.}

SUMARIO.

TEXTO: La primera sombrilla.- Mis grabaditos.

Cuentos: La lluvia del verano, La invasión, Un joven como hay muchos (fábula), Viajes de Gulliver.

$A R C A D E$ NOÉ. Incluye: refranes, pensamientos, sentencias, frases populares, Máximas, como las siguientes:

"Dá de comer al hambriento,

y Dios te dará sustento".

$* * *$

Quien alberga al peregrino

Del cielo encuentra el camino.

$* * *$

No hallarás un avariento

que esté tranquilo y contento.

En este mismo número se consigna la siguiente información relacionada con la impresión del periódico: "Tipografía de El Atomo Calle de La Libertad $\mathrm{N}^{\circ} 99 "$.

\section{$N^{\circ} 8: 15$ de setiembre de 1888}

SUMARIO.

TEXTO: ¡Perdón, Mamá!.- Mis Grabaditos.- Viajes de Gulliver.- El Pato y la Serpiente (fábulas).

ARCA DE NOÉ: (Dichos, máximas y refranes):

GRABADITOS.

\section{$N^{\circ}$ 9: 22 de setiembre de 1888}

SUMARIO.

TEXTO: ¡Perdón, Mamá!- Mis grabaditos.- A las niñas.- Amor filial (poesías).- Viajes de Gulliver.

ARCA DE NOÉ: "La ilusión y la realidad" (diálogo entre el camello y el caballo", a raíz de las protuberancias en la espalda del primero).

\section{$\mathrm{N}^{\circ} 10: 29$ de setiembre de 1888}

SUMARIO.

TEXTO: La luz misteriosa (cuento).- Mis grabaditos.- La Misión del maestro.- La inocencia (poesías).- El Ruiseñor (fábula).- Viajes de Gulliver.

ARCA DE NOÉ: 
Moralejas:

Máximas:

La honradez, bien soberano,

Mil disgustos ocasiona;

Pero al fin, tarde ó temprano

Alcanza digna corona.

Quien tiene caridad y alma pura;

De las faltas ajenas no murmura

En el templo no estés un solo instante

Sin una devoción edificante.

Cultura Infantil. Se fundó el 1 de junio de 1913, también en Trujillo, por Julio Eduardo Mannucci, quien dirigiera el Centro Escolar de Varones 241 "Pedro M. Ureña”, conocido como "Centro viejo". Se editaron 39 números, el último de ellos en noviembre de 1918, con un tiraje de 2000 ejemplares, con un precio de venta de $0.03 \mathrm{ctvs}$. el ejemplar. Entre sus principales colaboradores figuraban: Aparicio Castañeda, Alvaro de Bracamonte Orbegoso, César Vallejo, Fortunato Alva Saldaña, Carlos Vidal C., José Eulogio Garrido, Santiago Vallejo, Carlos C. Godoy, Víctor Alejandro Hernández, Antenor Orrego, Oscar Imaña, Alcides Spelucín, Federico Esquerre, Juan Espejo Asturrizaga, Francisco Xandóval; es decir, varios integrantes del famoso Grupo Norte. En esta revista publicó sus primeros poemas, casi todos ellos de carácter didáctico, el poeta César Vallejo, cuando se desempeñaba como profesor de la mencionada escuela. En efecto, Espejo Asturrizaga, en su libro "César Vallejo / Itinerario del hombre 1892-1923", anota la siguiente relación de poemas de Vallejo publicados en esta revista. ${ }^{1}$

"Fosforescencia", $\mathrm{N}^{\circ}$ 4, setiembre, 1913.

“Transpiración vegetal”, N 7, diciembre, 1913.

"Fusión", $\mathrm{N}^{\circ}$ 12, setiembre, 1914.

"Estival", $\mathrm{N}^{\circ} 23$, junio, 1916.

"El barco perdido", $\mathrm{N}^{\circ}$ 29, diciembre, 1916.

"Oscura", $\mathrm{N}^{\circ}$ 30, mayo, 26, 1917.

"La mula", $\mathrm{N}^{\circ}$ 32, julio, 1917.

“A mi hermano muerto", $\mathrm{N}^{\circ} 33$, agosto, 1917.

“Armada juvenil”, $\mathrm{N}^{\circ}$ 34, setiembre, 1917.

"Babel", N 37, diciembre, 1917. 
Hay que reiterarlo entones: el colaborador más notable llegó a ser César Vallejo, profesor de Ciencias del mencionado plantel, quien publicó una serie de poemas de tema y carácter didáctico, como "Fosforescencia", el primero que publicó el poeta, cuyo propósito era facilitar la comprensión de los temas lectivos a sus alumnos. Este poema fue publicado en el $\mathrm{N}^{\circ} 4$ de "Cultura Infantil”, en setiembre de 1917; "Transpiración vegetal “, en el No 7, en diciembre del mismo año. Después siguieron otros poemas del mismo carácter y propósito. Este hecho es harto ilustrativo para considerar al poeta santiaguino como uno de los primeros representantes de la poesía infantil y juvenil, aparte, por supuesto, de sus creaciones presentes en sus libros posteriores. Por lo expuesto, en el proceso de producción literaria, a la luz de la moderna teoría del texto y de la comunicación literaria, las primeras instancias están dominadas por el receptor y la adecuación pertinente del código al mensaje que se ha de construir y transmitir.

\section{DESARROLLO PANORÁMICO}

\section{Década del 40}

En los años siguientes se produce un largo vacío, que se prolonga hasta los albores de la década del 40, cuando Guillermo Ugarte Chamorro publica "Palomilla", que se editó entre 1940-45, habiendo llegado a editarse 42 números, con temas de carácter peruanista, junto a autores clásicos. Otros temas incidían en aspectos regionales y como ágil recurso técnico-gráfico, incorporó atractivas historietas. Sus lectores se distribuyeron por toda la geografía nacional.

\section{Década del 50}

En la década del 50 destaca la publicación de la revista ilustrada “Avanzada”, fundada en 1953 por Ricardo Durand Flores. El carácter principal de esta revista era el equilibrio entre los temas instructivos y otros relacionados con la identidad nacional. Impresa a colores y con circulación nacional, los relatos de aventuras eran realizados por tres personajes simbólicos representantes de costa, sierra y selva.

En 1958 Matilde Indacochea Pejovez funda “Juanito", importante y atractiva revista de contenido principalmente recreativo y didáctico, con textos sobre cuentos, poemas, adivinanzas, crucigramas e historietas. También se advertía la presencia de aspectos folclóricos y tradicionales de la realidad nacional. Esta revista se siguió editando hasta los primeros años de la siguiente década. 


\section{Década del 60}

En la década del 60 el proyecto más importante fue "Selecciones Escolares", excelente revista, que se publicó quincenalmente, bajo la dirección del maestro Antonio Olivas Caldas, acompañado de un notable equipo de maestros, escritores, periodistas y artistas gráficos, como la fina poetisa Esther M. Allison, y los intelectuales Emilio Delboy, Jaime López Raygada, Irene Silva Santolalla y otros distinguidos educadores y comunicadores de la época. La edición en colores y el excelente material fotográfico seguían un diseño parecido a la revista "Selecciones del Reader Digest”. El proyecto correspondió a la Central Peruana de Publicaciones; pero el contenido no solo fue literatura infantil, sino también historia, geografía, ciencia, tecnología, manualidades, humor, educación, etc. Los primeros números que se han registrado corresponden al año 1960. En conclusión, sin embargo, podemos afirmar que la revista "Selecciones Escolares", más que ubicarse en el ámbito de la literatura infantil y juvenil, corresponde a la literatura escolar por el marcado énfasis en la intención didáctica y pedagógica.

\section{Los años del 70 y la influencia de la Reforma de la Educación}

La década del 70, al influjo de la Reforma de la Educación Peruana alentada por el gobierno de la época, encabezado por Juan Velasco Alvarado, propicia la eclosión de publicaciones inspiradas y relacionadas con la niñez. Son los años en que se perfila y anuncia una revolución de materiales gráficos llamativos, coloridos e impactantes, manifestados en textos, láminas, colecciones, afiches, álbumes, material audiovisual, etc., que anuncian la revolución de la imagen que se iniciará en los próximos años. Como fenómeno consecuente de la expropiación de los medios periodísticos en el conjunto de otras reformas aplaudidas o cuestionadas, el diario "La Prensa" pasó a ser dirigida por Wálter Peñaloza quien, como parte de su labor al frente del mencionado medio periodístico, alentó y promovió la publicación de Urpi, revista semanal que se editaba como Suplemento Infantil, y que llegó a convertirse en el proyecto más ambicioso y exitoso de las revistas para niños. Empezó a publicarse a fines de 1974 y continuó en las siguientes semanas y meses del indicado año y de 1975. Como editora técnica actuó Gladys Padró, acompañada de una selecta plana de colaboradores, entre los que figuraron distinguidos escritores, investigadores, maestros, ilustradores y editores: Carlota Carvallo de Núñez, Charo Núñez de Patruco, Jaime Campodónico, Ada Bullón, José Luis Jordana Laguna, Jorge Bernuy, Isa Castro, Wilfredo Alvarado, Rosa Cerna Guardia, Mela Miranda, Patricio Andrade; mientras que en las áreas de diagramación y arte se advertía la presencia de: Lorenzo Osores, Nobuko, Jorge 
Eslava y José Zárate. El contenido primordial comprendía relatos, mitos, leyendas, poesía, creaciones textuales y dibujos a todo color por parte de niños de todo el país. Incluso varios de esos dibujos llegaron a ser temas de portada, junto a manualidades educativas y del hogar, relatos y vidas de personajes ilustres, temas de ciencia y tecnología, difusión de inventos, tiras cómicas, etc. En resumen, "Urpi" fue una revista literario-pedagógica.

En 1976 empieza a editarse "Creación", como suplemento de otro importante diario, también expropiado a sus antiguos propietarios: el diario "La Crónica”. Entonces la revista la publicaban Angel Avendaño y José Luque. Dicha publicación contenía dibujos y creaciones de niños, juegos y temas recreativos, junto a poemas y relatos de conocidos escritores. Editada en formato tabloide, con ilustraciones a colores, se convirtió en una de las revistas más entretenidas y amenas de su tiempo.

En 1978 apareció “Collera”, bajo la dirección de Myriam Balbuena y editada por el Centro de Estudios Sociales y Publicaciones. El contenido comprendía: cuento, poesía, leyendas, tradiciones, historias, curiosidades, amenidades y entretenimientos. También fueron frecuentes las tiras cómicas sobre sucesos, junto a notas informativas sobre determinados acontecimientos, e informaciones científicas.

Desde 1979 se edita la revista mensual "Aguilucho", dirigida por el padre Valentín García, bajo cuya dirección alcanza una excepcional continuidad y vigencia. Editada por el Centro de Animación Misionera, es una revista de orientación católica y actualmente es dirigida por el P. Miguel Villegas Serrano. Hasta ahora se han editado unos 350 números, caso sin precedentes en el país. Es una revista de excelente diseño, diagramación y edición. Contiene temas informativos, culturales y tradicionales del país y del extranjero. Una historieta permanente es los hechos y aventuras de Humonegro; sin embargo no son muy frecuentes los temas creativos propios de la literatura infantil y juvenil; tampoco se advierte la presencia de textos literarios de escritores adultos. Podríamos decir que se trata más bien de una revista escolar que bordea el ámbito de la literatura infantil sin pertenecer plenamente a dicho ámbito.

\section{Los años ochenta y la aparición de la APLIJ}

Los años ochenta constituyen una década singular y extraordinaria para el destino de la literatura infantil y juvenil en el país. A comienzos de esa década, el Instituto Nacional de Bienestar Familiar, por iniciativa de Roberto Rosario Vidal y el respaldo de Matilde Pérez Palacios, directora de la mencionada 
institución, convoca en Lima a varios poetas y narradores con producción para niños y jóvenes, cuyos textos fueron reunidos en "La barquita de papel"; a continuación empiezan a realizarse los primeros encuentros de escritores de literatura infantil. El punto de partida es la ciudad de Cajamarca. Como consecuencia inmediata se funda la Asociación Peruana de Literatura Infantil (APLI), que posteriormente ampliaría su cobertura a los jóvenes, por lo que la institución pasó a ser, como hasta ahora, la APLIJ.

En esta década se registra la aparición de las siguientes revistas:

- "La Mariposa de Cristal", dirigida por Roberto Rosario Vidal, dedicado a la difusión especial de determinados autores del género infantojuvenil, como Rosa Cerna Guardia, Jesús Cabel, Carlota Carvallo y el propio director. Solo se publicaron tres números: el $\mathrm{N}^{\circ} 1$ se publicó el 23 de diciembre de 1982 como suplemento de La Prensa y con un tiraje extraordinario de 42 mil ejemplares según declaró el propio Arturo Salazar Larraín, director tan importante diario; sin embargo, la revista se publicó con el título "Arca de papel”, que incluyó poemas de Cabel; el N² 2 apareció el 3 de enero de 1983; asimismo, con el título de "Víspera de reyes" se publicó un cuento de Carlota Carvallo de Núñez. El tiraje fue el mismo que en el primer caso. Ambas ediciones fueron posibles por el apoyo de Alicia Bustamante, esposa de Salazar Larraín. La ilustración de ambos números la realizó Jesús Rojas, también autor del logotipo de la APLIJ; en julio de 1983 se publicó el N 3, dedicado a Rosa Cerna Guardia. Incluyó "El girasol”, con ilustraciones de Maruja Naranjo, quien trabajaba en el Ministerio de Educación; actualmente es embajadora del Perú en Uruguay. Solo se imprimieron 500 números ${ }^{2}$.

- "La Chispita", dirigida por Zoila Loayza y editada por Víctor Manzur, con temas sobre relatos, formación pedagógica, historietas, recreación y cultura general. Sin embargo, no hemos podido precisar exactamente la fecha de fundación de esta revista.

- "Pequeña", publicación fundada en 1982 y elaborada por las niñas María Hortensia Apaza, Lourdes Graciela y Elvira, todas hijas del poeta Carlos Zúniga Segura. El contenido básicamente estaba constituido por lecturas seleccionadas por las propias editoras, lo cual revela el buen nivel estético y la importancia de la formación de la niñez desde el hogar. Por ejemplo, el número 3, estuvo conformado por textos poéticos de Carlos Oquendo de Amat, Carlos Augusto Salaverry, Livio Gómez, César Toro Montalvo, Carlos Zúñiga, Juan Gonzalo Rose y Carlos Meneses. 
- “Arco Iris", editada en Chiclayo desde 1982, bajo la dirección de Estuardo Deza Saldaña, Juan Sánchez y Víctor Díaz Monge. Incluye creaciones en poesía, cuento y comunicaciones para niños.

- “Cholito", revista editada desde 1982, en Chimbote, con el subtítulo "Revista Ilustrada para los Niños del Perú y América", bajo la dirección del escritor Oscar Colchado Lucio, radicado entonces en el puerto, donde estudió primaria, secundaria y superior. El nombre de la publicación está asociado al personaje creado por el autor para varias de sus obras narrativas. Precisamente, en el editorial del primer número se consigna expresamente: “¡Hola, queridos lectorcitos! Yo soy Cholito, un amiguito de ustedes. Conmigo trabajan en esta revistas buenos y entrañables amigos, como son el pelícano Pico largo, el Profe Pingüino, el Gato Cartero y tantos otros personajes de quienes ya conocerán más adelante. $^{3}$

Sin embargo, el proyecto concluyó después de unos pocos números de edición de la revista. Al parecer solo se editaron dos números.

- "Los niños del sol”, dirigida por Juan Rivera Palomino, fundada en 1983.

- "Visión Futuro". Excelente revista ilustrada, de periodicidad semanal, de tamaño tabloide, que se publicó en 1985, cuando empezó a publicarse el novedoso e impactante diario "Visión", primero bajo la dirección de César Hildebrandt; después, por Alfonso Reyes M. La edición, sin embargo, siempre correspondió a Danilo Sánchez Lihón, junto a quien figuraban: Víctor Escalante, como diseñador; Mercedes Ochoa, Carmela Sotomayor, Jorge Pimentel, como redactores; y Gredna Landolf, Charo Núñez y Nobuko Tadokoro, como ilustradores. Aunque fue una publicación dirigida a los niños y jóvenes, el contenido, no se limitó a la literatura infantil, sino también comprendió temas de ciencias naturales, historia, ciencia, tecnología y entretenimientos. En el campo literario se publicaron narraciones y poesía, tanto de autores consagrados como de los propios estudiantes. "Visión Futuro" también pertenece al ámbito de la literatura escolar.

- “Alboradita": Creación y análisis. Réplica de la revista mayor "Alborada", publicación oficial del importante Grupo de Arte y Literatura "Isla Blanca", de intensa actividad cultural en la región. El primer número de "Alboradita" apareció en 1985, bajo la responsabilidad editorial de Gonzalo Pantigoso, Félix Ruiz Suárez y Carmen Mimbela. Todos los números de la revista, al igual que "Alborada" son de naturaleza estrictamente literaria en poesía, narración y ensayo. Sin embargo, el esfuerzo ya no se ha continuado. 
- “La Gota", revista breve, de formato muy ágil, fundada en 1986, en Chiclayo, por el poeta Enrique Solano Rodríguez. Su contenido está constituido primordialmente por creaciones poéticas de escritores adultos peruanos y extranjeros ganados por la inspiración infantil. Los primeros números, sin embargo, se publicaron en Lima. Sin periodicidad fija o regular, se han editado varios números.

\section{Década del 90}

- “Creadores". Esmerada e importante revista de teoría, ensayo, crítica y pedagogía, fundada en 1997, dirigida en Huaraz por los escritores e intelectuales Carlos Toledo Quiñónez y Néstor Espinoza, y complementada con textos poéticos de reconocidos escritores ancashinos, como: Carlos Eduardo Zavaleta, Román Obregón Figueroa, Oscar Colchado Lucio, Francisco González, Elmer Moreno Martel, entre otros.

- “Candil”. Revista de la Asociación Peruana de Literatura Infantil y Juvenil, filial Jaén, aparecida el 5 de mayo de 1999, cuando ejercía la Presidencia de la APLIJ-Jaén Wilson Baca Altamirano. "El origen del nombre surge al compararse con los fines de la APLIJ. Valgan verdades, hasta el año 99 nuestra institución apenas era conocida por algunos sectores de la población. La timidez y la inercia eran los signos de aquellos tiempos. ${ }^{4}$ No tiene periodicidad fija, pero ya lleva publicados varios números. La revista se edita ahora bajo la dirección del arqueólogo y promotor cultural Ulises Gamonal Guevara, a quien acompaña un importante grupo de colaboradores, como: Cesarina Vásquez Torres, Doris Carranza Gálvez, José Lenin Sánchez Ramos, Maritza Chávez Avalos, Paulina Jiménez de Nava, Ernesto Arias Díaz, Virginia Vásquez Torres, Teófilo Gutiérrez, Tomás Paredes Díaz, Segundo Villanueva Alarcón, etc., quienes desarrollan un esmerado trabajo educativo, literario y cultural. En el contenido predominan textos de ensayo, crítica y creación.

\section{EL SIGLO XXI}

- "Poroporo". Revista de Literatura Infantil y Promoción de la Lectura, fundada y dirigida en Lambayeque por el reconocido escritor y maestro Javier Villegas Fernández. Se fundó en el año 2001. Los textos pertenecen a escritores adultos ganados por la inspiración de la niñez.

- "Pastorala". Revista de la APLIJ, filial en Cajamarca, fundada en agosto del 2003, dirigida por el escritor Luzmán Salas Salas, ex Presidente de 
la APLIJ y el más autorizado conocedor de la literatura de su región. El Consejo de Redacción está constituido por Camilo Terrones Cotrina, Ricardo Cabanillas Aguilar, Dalila Paredes Canto, Isabel Castañeda de Cabanillas, Rosa Reaño Tirado y Wilson Ocharán Sánchez. Incluye temas de teoría literaria, didáctica de la literatura infantil, el cuento y la declamación, así como creaciones de niños y jóvenes. El nombre de la novedosa y sugerente revista remite inmediatamente a la creación del notable poeta andinista y para la infancia Mario Florián. Así lo expresa textualmente el director en el primer número: "Orgullosos de nuestro patrimonio literario cajamarquino, inspirados en el ritmo poético de nuestro insigne vate Mario Florián, entregamos nuestra palabra bajo el título de PASTORALA, mensajera fraterna nutrida de pensamiento y sentimiento andinos, en la ruta que nos lleva a encontrarnos con las hermanas filiales de todos los rincones de la patria peruana".

- Del 2005 o 2006 es "La barca de eucalipto", dirigida por Dios Dado, cuyo segundo número, registra su nota editorial en abril del 2006, con la mayor parte de autores pertenecientes a la región La Libertad; sin embargo, en la página "de créditos" aparece la indicación "Hecho e impreso en Piura; incluso, allí mismo se consigna que la revista es editada por el Fondo Editorial Santa Angela, de Piura. Es una revista de creación poética y narrativa de escritores adultos (aparte del autor, aparecen: Jorge Barboza, Marleni Carranza Sandoval, Alejandro Benavides, Carlos Sánchez Vega y Luis Cabrera Vigo. Entre los piuranos figuran: José María Gahona, Margarita Cori Retamozo, Gilberto Vegas, Alberto Alarcón y Kiko Gonzales.

- “Cuculí”. Revista de literatura infantil y juvenil, de Tacna, también fundada a comienzos de la primera década del presente siglo, aproximadamente el año 2008. Se edita bajo la dirección de Gabriela Caballero Delgado. La edición está a cargo de William González Huanacune. La relación de colaboradores la han venido integrando: Luis Alberto Calderón Albarracín, Luis Chambilla Herrera, Katherine Estrella Palacios, Guido Fernández de Córdoba, Livio Gómez, André González Caballero, Miler Huanca, Edita Luyo, Paola Mamani, Esperanza Martínez, Jhon Ortega, Reynaldo Palacios, Guillermo Quintanilla, Omar Silbert Salas y Artidoro Velapatiño.

- "Fabulador". Revista de literatura infantil y juvenil, editada en la Universidad Nacional Mayor de San Marcos por un Comité Editor integrado por: Miguel Angel Carhuaricra Anco, Jhoana Herrera Fabián y Karen Huachaca Avendaño, bajo la asesoría de los profesores Mauro Mamani Macedo y Rosa Carbonel Polo. El primer número apareció en octubre del 2012. El fin que 
persigue la revista está expresado en la nota editorial del primer número: “difusión de artículos, ensayos y entrevistas, así como reseñas y comentarios que contribuyan a la promoción y animación de la lectura en los primeros años”. En concordancia con dicha finalidad, "la revista está dirigida a docentes, investigadores, especialistas y todo aquel cuyo interés sea la literatura para pequeños lectores; por ello no se orienta solo a la creación, sino también a la crítica y teorización de este tipo de literatura". ${ }^{6}$

La estructura de la revista comprende secciones originalmente tituladas con denominaciones asociadas al nombre: "Fabulistas", con inclusión de ensayos y artículos; "Entrefabuladores", o entrevistas; "Fabulaciones", de creación literaria (cuento y poesía para niños y jóvenes); "Fabulecturas", que consiste en las reseñas o críticas de libros; y "Travesía fabuladora", referida a recomendaciones sobre páginas Web, teatro, bibliotecas y eventos sobre literatura infantil y juvenil. La revista es de esmerada, novedosa e importante elaboración y contenido. El segundo número se publicó en octubre del 2013.

- “Para leerte mejor". También lleva el subtítulo "Revista de Literatura Infantil y Juvenil”. El primer número apareció en julio del 2012, producida por el siguiente equipo: director, Erick López Sánchez; directora académica, Irina Burgos Chorres; diseño y diagramación, Gabriel Valladares Valiente; ilustración, Marco Pariona López; corrector de estilo, Andrea Díaz Bravo. Es una publicación de contenido teórico, analítico, crítico y de propuestas didácticas, nacida bajo el influjo del fenómeno de los planes lectores, como se indica en el propio editorial: "En el panorama actual, tanto editorial como escolar, la LIJ se ha convertido en protagonista. Esto ha ocurrido de manera vertiginosa por la conformación de los planes lectores en las escuelas públicas y privadas, donde muchas veces este tipo de literatura todavía es vista con misterio, pero también como un arte menor".

- Otra revista aparecida en Lima, también en 1912, es "Pristina: revista infantil de creación literaria"; sin embargo, no tenemos mayor información. Casi lo mismo se puede decir de la revista editada en versión electrónica “Caballito de madera", bajo la dirección de Vicky Canales.

\section{4. "RAYUELO" Y LA APLIJ}

En el contexto así expuesto adquiere sentido e importancia la revista "RAYUELO", órgano oficial de la Asociación Peruana de Literatura Infantil y Juvenil (APLIJ). En efecto, esta organización, fundada en 1983, agrupa a los 
escritores, investigadores, promotores, editores, comunicadores y docentes cuyo signo común es el ejercicio de la literatura para niños y jóvenes.

Desde el año de su fundación, sin fallar ninguno, nos reunimos puntualmente en nuestros Encuentros Nacionales y también Internacionales en diferentes ciudades del Perú. Nos hemos congregado en Cajamarca, Tumbes, Sullana, Chiclayo, Trujillo, Huaraz, Lima, Ica, Huancayo, Tarma, Huancavelica, Ayacucho, Paramonga, Arequipa, Cuzco, Tacna, Huánuco, Tingo María, Jaén... Sin vanagloria, aunque los participantes son en número variado en cada ocasión convocamos a varias decenas y centenas de participantes. Incluso en algunas ocasiones hemos sobrepasado el millar y medio de asistentes.

\section{Fundación}

Pero para no desviarnos del tema, Rayuelo apareció por primera vez en marzo de 1995, en Chimbote, bajo los auspicios de la Universidad Nacional del Santa $^{8}$, cuando el autor del presente trabajo asumió la Presidencia de la APLIJ el año anterior. Este es un hecho histórico y extraordinario para nuestro movimiento, porque por fin concretábamos la aspiración de contar con nuestra propia revista, aunque, en verdad, el primer número apareció simplemente como Boletín. El formato y estructura de revista se determinó a partir del segundo número.

Pero, la naturaleza y carácter primordial del contenido; es decir, lo que le otorga personalidad propia, es que, curiosamente, no es una revista dirigida o destinada principalmente a los niños, sino a los adultos, pues somos conscientes de que, en un país como el nuestro, de precarios índices de lectura formativa, de maestros necesitados de una permanente orientación y actualización, de la posibilidad de aprovechar los aportes teóricos y críticos del más alto nivel, a quienes hay que formar es a los formadores, trátese de maestros, escritores o promotores.

\section{Contenido}

Según lo expuesto, el contenido primordial de "Rayuelo" está constituido por temas de investigación, ensayo y crítica; de enfoques y planteamientos teóricos, metodológicos y didácticos; de análisis y comentario de libros; de exposición de experiencias y planteamientos; reseñas de publicaciones (libros y revistas); entrevistas y crónicas. Asimismo, como cada Encuentro de la APLIJ lleva el nombre de un escritor emblemático, cada número de Rayuelo desarrolla temas de homenaje al respectivo escritor, en la línea de revaloración, actualización, permanencia y propagación de su obra literaria y magisterial. 
Por eso, a través de los sucesivos números, hemos dedicado secciones de homenaje a escritores notables, pero no siempre avivados por el fuego de la memoria. Entonces, en una visión de conjunto, nuestra revista, al unísono con los Encuentros, ha dedicado sus páginas a exaltar la memoria y la obra de escritores que consagraron su inspiración e inquietud a la formación de los niños y jóvenes de nuestra patria y de más allá de nuestras fronteras. Allí están las páginas dedicadas a Luis Valle Goicochea, Abraham Arias Larreta, Enrique López Albújar, Iván Tello Carbajal, Mariano Melgar, Abraham Valdelomar, Omar Zilbert Salas, Orfelinda Herrera de Angeles, Rosa Cerna Guardia, Catalina Recavarren, Manuel Ibáñez Rosazza, Teófilo Maguiña Cueva, Ricardo Palma, Francisco Izquierdo Ríos, Eduardo de la Cruz Yataco, Mario Florián, Ciro Alegría, José María Arguedas, Gerardo de Gracia, Julio Garrido Malaver, etc.

En otras ocasiones, "Rayuelo" ha dedicado artículos y ensayos acerca de la personalidad y producción literario-educativa de distinguidos miembros de la APLIJ, como Iván Tello Carbajal y Eduardo de la Cruz Yataco, de Lima; Marcial Molina Richter, de Ayacucho; Arnaldo Panaifo Teixeira, de Iquitos; y Luis Alberto Calderón, de Tacna.

\section{Autores y colaboradores}

Entre los autores y colaboradores de artículos destacan escritores de unánime prestigio y reconocimiento, varios de ellos con matices creativos en materia de literatura infantil y juvenil no muy conocidos o poco difundidos. Así lo constituyen los textos de: Ciro Alegría, Javier Sologuren, Luis Alberto Sánchez, Jorge Eslava, César Toro Montalvo, María Teresa Pollarolo, Rosa Cerna Guardia, Ismael Pinto, Manuel Velásquez Rojas, Antonio Cornejo Polar, Jorge Díaz Herrera.

Entre los miembros de la APLIJ cuyos textos continuamente otorgan presencia y personalidad a "Rayuelo" figuran: Jesús Cabel Moscoso, Roberto Rosario Vidal, Manuel Ibáñez Rosazza, Ruth Miranda Villena, Danilo Sánchez Lihón, Eduardo de la Cruz Yataco, Oscar Espinar La Torre, Lilibeth Felices Morales, Marcial Molina Richter, Luis Alberto Calderón, Gaby Arce Muñoz, Aureo Sotelo Huerta, Luzmán Salas Salas.

Por otro lado, la creciente importancia que viene alcanzando "Rayuelo" tanto en el ámbito nacional como internacional ha determinado la recepción de artículos procedentes de varios países, en cuyo conjunto merecen destacarse las colaboraciones de Luis Cabrera Delgado y Yaima Rodríguez, de Cuba; Sylvia Puentes de Oyenard, de Uruguay; Catalina Guerrero, de Madrid; Armando José Sequera, de Venezuela. 


\section{Edición}

La edición de Rayuelo es un trabajo integrado por varios miembros de nuestra organización, y por gente amiga, como: Jesús Cabel, Roberto Rosario Vidal, Oscar Espinar, Aníbal Paredes Galván, Bety Sánchez Layza, Eliodoro Martínez Suárez, Carlos Sánchez Vega, Luzmán Salas Salas, Jackeline Ulloa.

\section{Valor educativo y cultural}

Por otro lado, el valor e importancia que viene alcanzando Rayuelo en el contexto de la educación y la cultura peruana puede resumirse en los siguientes hechos:

a) Contribución al esclarecimiento y definición de la naturaleza y modo de ser de la literatura infantil y juvenil.

b) Medio de difusión de las creaciones literarias de escritores, tanto consagrados como aquellos urgidos de un espacio axiológico y difusor.

c) Medio de información teórica, cognoscitiva y didáctica para el magisterio nacional.

d) Medio de difusión de las expresiones literarias locales, regionales, nacionales e internacionales.

e) Medio de valoración y difusión de la cultura tradicional, el folclore y el relato de tradición oral.

f) Orientación didáctica y metodológica para el magisterio dedicado a la formación de la niñez y juventud de nuestra patria.

\section{CONCLUSIONES}

a) Los antecedentes más lejanos de las revistas de literatura infantil y juvenil lo constituyen "El Átomo" y "Cultura Infantil”, ambos aparecidos en Trujillo: el primero, en 1888; el segundo, en 1913.

b) Las revistas de literatura infantil de mayor permanencia son: "Cultura infantil”, dirigida por Julio Eduardo Mannucci, con 39 números editados entre 1913 y 1918; "Palomilla", con 42 números, editada de 1940 a 45, por Guillermo Ugarte Chamorro; “Urpi”, con 47 números, entre los años 1974-75, bajo la dirección de Wálter Peñaloza: y "Rayuelo", la de mayor duración, desde 1995 hasta el presente, con 21 números. Puede agregarse también "Collera", con 10 números, que circuló entre 1978 y 1979.

c) Casi todas las revistas priorizan los textos creativos, tanto de escritores adultos, muchos consagrados y reconocidos, con las creaciones propias de los niños. Pocas son las revistas destinadas a la formación teórica, crítica, de análisis e investigación. 
d) Las diferentes revistas corresponden a proyectos personales, de pequeños grupos, organismos o empresas editoriales.

e) La gran mayoría de revistas son realizadas por escritores, maestros, editores, diseñadores adultos orientados a la formación de la niñez. En ese conjunto, la edición de "Pequeña", realizada por las entonces niñas, las hermanas Zúñiga-Apaza, es un caso singular, aunque también hay que reiterarlo: requirieron la orientación de su padre, el escritor Carlos Zúñiga Segura.

f) Si bien la mayoría de revistas pueden considerarse como medios de estímulo, difusión y creación de la literatura infantil y juvenil, varias otras, como "Selecciones Escolares" y "Aguiluchos", de gran importancia educativa y cultural, se inscriben con mayor propiedad en el ámbito de la "literatura escolar" por su énfasis didáctico y formativo.

g) "Rayuelo" es la única revista perteneciente a una institución orgánicamente constituida y funcional, como la APLIJ.

h) La edición de "Rayuelo" permite a la APLIJ propagar la memoria y la obra de varios de los escritores regionales, nacionales y también internacionales, especialmente aquellos bajo cuyo nombre se desarrollan los sucesivos encuentros nacionales e internacionales de nuestra organización.

i) "Rayuelo" prioriza intencionalmente su contenido con temas de historia, teoría, crítica, análisis y reseña de publicaciones. Está dirigida principalmente a los adultos en cuanto responsables de la formación de las nuevas generaciones.

\section{NOTAS}

* Texto de la conferencia desarrollada en el Congreso Nacional de Literatura Infantil y Juvenil realizada por la Casa de la Literatura en mayo del 2014.

1 Espejo Asturrizaga, Juan. César Vallejo: Itinerario del hombre 1892-1923. Lima, Seglusa Editores, 1989, p. 217.

2 Información brindada por Roberto Rosario Vidal mediante correo electrónico. 13/4/2014.

3 Nota editorial del $\mathrm{N}^{\circ} 1$. Chimbote, abril, 1982, p. 2.

4 Nota editorial del $\mathrm{N}^{\circ}$ 5, Jaén, noviembre, 2005, p.2

5 Nota de la Presentación. Cajamarca, agosto, 2003, p.2.

6 Lima, 2012, $\mathrm{N}^{\circ} 1$, p.3.

7 Lima, julio, 2012, p. 3.

8 Como al mismo tiempo, el autor del artículo ejercía el rectorado de la Universidad Nacional del Santa, encargamos la edición del primer número de "Rayuelo" al escritor Gonzalo Pantigoso Layza, profesor de la mencionada institución. 